\title{
Respiratory System Link Identifier
}

National Cancer Institute

\section{Source}

National Cancer Institute. Respiratory System Link Identifier. NCI Thesaurus. Code C162025.

A sequence of characters used as a linkage between related respiratory system findings identifier records. 